\title{
THE EVOLUTION OF MOMENT GENERATING FUNCTIONS FOR THE WRIGHT-FISHER MODEL OF POPULATION GENETICS
}

\author{
TAT DAT TRAN, JULIAN HOFRICHTER, JÜRGEN JOST
}

\begin{abstract}
We derive and apply a partial differential equation for the moment generating function of the Wright-Fisher model of population genetics.
\end{abstract}

\section{Contents}

1. Introduction

2. Master equation

2.1. 2 alleles

2.2. $K+1$ alleles

3. Moment generating functions

3.1. 2 alleles

3.2. $K+1$ alleles

Acknowledgement

References

MSC2000: 60J27, 60J60

Key words: Wright-Fisher model, random genetic drift, moment generating function, master equation

\section{INTRODUCTION}

The Wright-Fisher model, that is, the random genetic drift model developed by Fisher [2] and by Wright [12] and mathematically solved by Kimura [6, 7] is the basic stochastic model in population genetics (see for instance [1]). The discrete model is concerned with the evolution of the probabilities between non-overlapping generations in a population of fixed size of two or more alleles obtained from random sampling in the parental generation. This basic model thus describes random genetric drift, and additional biological mechanisms like mutation, selection, or a spatial population structure can then be superposed. As such, the model works with a finite population in discrete time, but the mathematical analysis of Kimura and others

Date: October 4, 2018. 
turned to its diffusion approximation suggested by Kolmogorov. This diffusion approximation works with an infinite population in continuous time. It consists of two partial differential equations of parabolic type for the probability density function for the various alleles, the so-called forward and backward Kolmogorov equations. In [10, 11, we have presented a general solution scheme for the associated diffusion process that keeps track of the population across possible allele losses. With our scheme, all basic quantities of interest, like expected times of allele losses, can be readily derived. In other words, we derive a global solution, in contrast to the local ones of Kimura and others. A crucial ingredient in our scheme are the equations for the moments of the probability distribution.

A somewhat simpler model than the Wright-Fisher model that however leads to the same diffusion approximation is the Moran model, a simple birth-death process in continuous time, see [1]. In the Wright-Fisher model, when creating the next generation, for each new member of the population a parent in the previous generation is randomly chosen. This has the consequence that one and the same individual in that parent generation could produce several offspring. In the Moran model, in contrast, a randomly chosen individual gives birth to a clone, and then another random individual in the population is killed to keep the population size constant. Thus, here, in each step, only one offspring is produced.

As mentioned, the Kolmogorov diffusion equations are concerned with asymptotic quantities, and in particular, do not account for small population size effects. Therefore, Houchmandzadeh and Vallade [4] have proposed to use the master equation for the probability distribution to directly derive a partial differential equation for the probability generating function of the process. This approach can produce exact formulae even for finite populations. In [4, this has been carried out for the Moran model with two alleles, not only for the basic model of random genetic drift, but also including the case of selection.

In the present paper, we derive a partial differential equation for the exponential moment generating function of the Wright-Fisher model with arbitrarily many alleles, utilizing our scheme of moment equations mentioned above. From this scheme, we can then also easily rederive formulae for quantities of interest like fixation probabilities. In order to facilitate the understanding, we shall always first treat the simplest case of two alleles and then present the case of arbitrarily many alleles.

\section{Master EQUATion}

In this section we shall use the master equations that express the evolution of the probability distribution for the alleles in the population in terms of the transition probabilities, in order to derive differential equations for the moments of the process. 
2.1. 2 alleles. Consider a continuous time stochastic process $\left\{X_{t}\right\}_{t \geq 0}$ with values in

$$
S_{1}^{2 N}=\left\{0, \frac{1}{2 N}, \ldots, 1\right\}
$$

with transition rates $B(k, j)$ from state $k$ to $j$ specified below.

The master equation for the probability function

$$
P(t, i, j)=\mathbb{P}\left(X_{t}=\frac{j}{2 N} \mid X_{0}=\frac{i}{2 N}\right)
$$

will be

$$
\frac{\partial P(t, i, j)}{\partial t}=\sum_{k=0}^{2 N} P(t, i, k) B(k, j), \quad \forall i, j=\overline{0,2 N}
$$

with the initial values $P(0, i, j)=\delta_{i j}$. In matrix form, this reads as

$$
\left\{\begin{array}{l}
\frac{\partial P(t)}{\partial t}=P(t) B, \quad \forall t \geq 0 \\
P(0)=I
\end{array}\right.
$$

This is a linear problem (2.2), with the unique solution $P(t)=e^{B t} . B$ and $P(t)$ then commute, and therefore we also have

$$
\frac{\partial P(t, i, j)}{\partial t}=\sum_{k=0}^{2 N} P(t, k, j) B(i, k), \quad \forall j=\overline{0,2 N} .
$$

For $B$, we consider two cases

$$
\sum_{k=0}^{2 N}\left(\frac{k}{2 N}\right)^{n} B(i, k)=\frac{n(n-1)}{2}\left(\left(\frac{i}{2 N}\right)^{n-1}-\left(\frac{i}{2 N}\right)^{n}\right), \quad \forall n \geq 0 ; i=\overline{0,2 N}
$$

and

$$
B(i, k)=\left(\begin{array}{c}
2 N \\
k
\end{array}\right)\left(\frac{i}{2 N}\right)^{k}\left(1-\frac{i}{2 N}\right)^{2 N-k}-\delta_{i, k}, \quad i, k=\overline{0,2 N}
$$

Remark 2.1. - Case (2.5) corresponds to the Wright-Fisher model, more precisely a continuous time Wright-Fisher model with discrete states, whereas the implicit scheme (2.4) will get rid off certain error terms for finite population size.

- In (2.4), which has been directly constructed from the moment equation, the coefficients $B(i, k)$ could become negative (see for example [3] for such a generalization of the master equation concept).

- Since in general the transition rates $B(i, j)$ are nonzero for any pair $i, j$, in our master equations, starting from state $\mathbf{i}$ we can directly access any other state with positive probability. Therefore, for the probability generating function, we would get an $2 N$-order partial differential equation which may be hard to solve. In contrast, for 
the moment generating function, we shall get a second order partial differential equation which can be solved by a simple expansion.

We shall prove that in the limit of the population size $(2 N \rightarrow \infty)$, these master equations will produce the classical Wright-Fisher diffusion equations. Moreover we shall prove that these master equations satisfy the moment formulae, exactly ofr (2.4) and approximately for (2.5). Therefore we can apply the moment generating function technique to calculate the conditional probability function.

In fact, when $2 N$ is sufficient large, we set

$$
x=\frac{i}{2 N}, \quad y=\frac{j}{2 N}, \quad z=\frac{k}{2 N}, \quad d x=d y=d z=\frac{1}{2 N}
$$

and

$$
p(t, x, y) d y=P(t, i, j), \quad b(x, y)=B(i, j) .
$$

Then, we obtain from (2.3)

$$
\frac{\partial p(t, x, y)}{\partial t}=\sum_{z} p(t, z, y) b(x, z), \quad \forall y \in S_{1}^{2 N},
$$

Now we expand the function $p(t, z, y)$ in $z$ at $x$ and obtain

$$
p(t, z, y)=\sum_{n \geq 0} \frac{1}{n !} \frac{\partial^{n} p(t, x, y)}{\partial x^{n}}(z-x)^{n} .
$$

(1) In the case of (2.4), we obtain

$$
\sum_{z} z^{n} b(x, z)=\frac{n(n-1)}{2}\left(x^{n-1}-x^{n}\right), \quad \forall n \geq 0 ; x \in S_{1}^{2 N} .
$$

It follows by induction that

$$
\left\{\begin{array}{l}
\sum_{z}(z-x)^{n} b(x, z)=0, \quad n \neq 2 \\
\sum_{z}(z-x)^{2} b(x, z)=x(1-x) .
\end{array}\right.
$$

Then we obtain

$$
\begin{aligned}
\frac{\partial p(t, x, y)}{\partial t} & =\sum_{z} p(t, z, y) b(x, z) \\
& =\sum_{z}\left\{\sum_{n \geq 0} \frac{1}{n !} \frac{\partial^{n} p(t, x, y)}{\partial x^{n}}(z-x)^{n}\right\} b(x, z) \\
& =\frac{x(1-x)}{2} \frac{\partial^{2} p(t, x, y)}{\partial x^{2}}
\end{aligned}
$$

which is exactly the classical Wright-Fisher diffusion equation.

(2) In the case of (2.5), we obtain

$$
\sum_{z} z^{n} b(x, z)=\frac{n(n-1)}{4 N}\left(x^{n-1}-x^{n}\right)+O\left(\frac{1}{N^{2}}\right), \quad \forall n \geq 0 ; x \in S_{1}^{2 N} .
$$


It follows by induction that

$$
\left\{\begin{array}{l}
\sum_{z}(z-x)^{n} b(x, z)=O\left(\frac{1}{N^{2}}\right), \quad n \neq 2 \\
\sum_{z}(z-x)^{2} b(x, z)=\frac{x(1-x)}{2 N} .
\end{array}\right.
$$

Then we obtain

$$
\begin{aligned}
\frac{\partial p(t, x, y)}{\partial t} & =\sum_{z} p(t, z, y) b(x, z) \\
& =\sum_{z}\left\{\sum_{n \geq 0} \frac{1}{n !} \frac{\partial^{n} p(t, x, y)}{\partial x^{n}}(z-x)^{n}\right\} b(x, z) \\
& =\frac{x(1-x)}{4 N} \frac{\partial^{2} p(t, x, y)}{\partial x^{2}}+O\left(\frac{1}{N^{2}}\right)
\end{aligned}
$$

which is an approximation of the Wright-Fisher diffusion equation.

Now we shall use the master equation (2.1) to derive the moment equation.

In fact, the $n$-th moment of this conditional probability function is

$$
m_{n}(t):=\sum_{j=0}^{2 N}\left(\frac{j}{2 N}\right)^{n} P(t, i, j) .
$$

Therefore we have

$$
\begin{aligned}
\dot{m}_{n}(t)= & \sum_{y} y^{n} \frac{\partial p(t, x, y)}{\partial t} \frac{1}{2 N} \\
= & \sum_{y} y^{n} \sum_{z} p(t, x, z) b(z, y) \frac{1}{2 N} \quad \text { by the Master equation (2.1) } \\
= & \sum_{z}\left(\sum_{y} y^{n} b(z, y)\right) p(t, x, z) \frac{1}{2 N} \\
= & \sum_{z} \frac{n(n-1)}{2}\left(z^{n-1}-z^{n}\right) p(t, x, z) \frac{1}{2 N} \quad \text { by the formula for } b \text { from (2.4) } \\
& \left(=\sum_{z} \frac{n(n-1)}{4 N}\left(z^{n-1}-z^{n}\right) p(t, x, z) \frac{1}{2 N}+O\left(\frac{1}{N^{2}}\right) \quad \text { by the formula for } b \text { from (2.5) }\right) \\
= & -\frac{n(n-1)}{2} m_{n}(t)+\frac{n(n-1)}{2} m_{n-1}(t) \quad \text { in the case of (2.4) } \\
& \left(=-\frac{n(n-1)}{4 N} m_{n}(t)+\frac{n(n-1)}{4 N} m_{n-1}(t)+O\left(\frac{1}{N^{2}}\right) \quad \text { in the case of (2.5) }\right) .
\end{aligned}
$$


2.2. $K+1$ alleles. Consider a continuous time stochastic process $\left\{\mathbf{X}_{t}\right\}_{t \geq 0}$ with values in

$S_{K}^{2 N}:=\left\{\frac{\mathbf{i}}{2 N}=\left(\frac{i_{1}}{2 N}, \cdots, \frac{i_{K}}{2 N}\right): i_{u} \in \mathbb{N}_{0}\right.$ for all $u=\overline{1, K}$, and $\left.\sum_{u=1}^{K} i_{u} \leq 2 N\right\}$.

To simplify the notation, we also put

$\Omega_{K}^{2 N}:=2 N S_{K}^{2 N}=\left\{\mathbf{i}=\left(i_{1}, \cdots, i_{K}\right): i_{u} \in \mathbb{N}_{0}\right.$ for all $u=\overline{1, K}$, and $\left.\sum_{u=1}^{K} i_{u} \leq 2 N\right\}$.

The master equation for the conditional probability function

$$
P(t, \mathbf{i}, \mathbf{j})=\mathbb{P}\left(\mathbf{X}_{t}=\frac{\mathbf{j}}{2 N} \mid \mathbf{X}_{0}=\frac{\mathbf{i}}{2 N}\right)
$$

then is

$$
\frac{\partial P(t, \mathbf{i}, \mathbf{j})}{\partial t}=\sum_{\mathbf{k} \in \Omega_{K}^{2 N}} P(t, \mathbf{i}, \mathbf{k}) B(\mathbf{k}, \mathbf{j}), \quad \forall \mathbf{i}, \mathbf{j} \in \Omega_{K}^{2 N},
$$

with initial values $P(0, \mathbf{i}, \mathbf{j})=\delta_{\mathbf{i j}}$. In matrix form, we have

$$
\left\{\begin{array}{l}
\frac{\partial P(t)}{\partial t}=P(t) B, \quad \forall t \geq 0 \\
P(0)=I
\end{array}\right.
$$

As for 2 alleles, the problem (2.12) has a unique solution $P(t)=e^{B t}$, and $B$ and $P(t)$ commute, and therefore we also have

$$
\frac{\partial P(t, \mathbf{i}, \mathbf{j})}{\partial t}=\sum_{\mathbf{k} \in \Omega_{K}^{2 N}} P(t, \mathbf{k}, \mathbf{j}) B(\mathbf{i}, \mathbf{k}), \quad \forall \mathbf{i}, \mathbf{j} \in \Omega_{K}^{2 N},
$$

where $B$ is defined by the formulae

$$
\begin{array}{r}
\sum_{k \in \Omega_{K}^{2 N}}\left(\frac{\mathbf{k}}{2 N}\right)^{\boldsymbol{\alpha}} B(\mathbf{i}, \mathbf{k})=-\frac{|\boldsymbol{\alpha}|(|\boldsymbol{\alpha}|-1)}{2}\left(\frac{\mathbf{i}}{2 N}\right)^{\boldsymbol{\alpha}}-\sum_{u=1}^{K} \frac{\alpha_{u}\left(\alpha_{u}-1\right)}{2}\left(\frac{\mathbf{i}}{2 N}\right)^{\boldsymbol{\alpha}-e_{u}}, \\
\forall \boldsymbol{\alpha} \in \mathbb{N}_{0}^{K} ; \mathbf{i} \in \Omega_{K}^{2 N} .
\end{array}
$$

We shall prove that in the limit of the population size $(2 N \rightarrow \infty)$, this master equation will yield the Wright-Fisher diffusion equation. Again, this master equation will satisfy the moment formulae and we can apply the moment generating function technique to calculate the conditionalal probability function.

In fact, when $2 N$ is sufficient large, we set

$$
\mathbf{x}=\frac{\mathbf{i}}{2 N}, \quad \mathbf{y}=\frac{\mathbf{j}}{2 N}, \quad \mathbf{z}=\frac{\mathbf{k}}{2 N}, \quad d x_{u}=d y_{u}=d z_{u}=\frac{1}{2 N}
$$

and

$$
p(t, \mathbf{x}, \mathbf{y}) \frac{1}{2 N}=P(t, \mathbf{i}, \mathbf{j}), \quad b(\mathbf{x}, \mathbf{y})=B(\mathbf{i}, \mathbf{j}) .
$$


Then, we obtain from (2.13)

$$
\frac{\partial p(t, \mathbf{x}, \mathbf{y})}{\partial t}=\sum_{\mathbf{z}} p(t, \mathbf{z}, \mathbf{y}) b(\mathbf{x}, \mathbf{z}), \quad \forall \mathbf{y} \in S_{K}^{2 N}
$$

Now we expand the function $p(t, \mathbf{z}, \mathbf{y})$ in $\mathbf{z}$ at $\mathbf{x}$ to obtain

$$
p(t, \mathbf{z}, \mathbf{y})=\sum_{\boldsymbol{\alpha}} \frac{1}{\boldsymbol{\alpha} !} \frac{\partial^{\boldsymbol{\alpha}} p(t, \mathbf{x}, \mathbf{y})}{\partial \mathbf{x}^{\boldsymbol{\alpha}}}(\mathbf{z}-\mathbf{x})^{\boldsymbol{\alpha}} .
$$

We obtain from the formulae (2.14) that

$$
\sum_{\mathbf{z}} \mathbf{z}^{\boldsymbol{\alpha}} b(\mathbf{x}, \mathbf{z})=-\frac{|\boldsymbol{\alpha}|(|\boldsymbol{\alpha}|-1)}{2} \mathbf{x}^{\boldsymbol{\alpha}}+\sum_{u=1}^{K} \frac{\alpha_{u}\left(\alpha_{u}-1\right)}{2} \mathbf{x}^{\boldsymbol{\alpha}-e_{u}}, \quad \forall \boldsymbol{\alpha} \in \mathbb{N}_{0}^{K} ; \mathbf{x} \in S_{K}^{2 N}
$$

It follows by induction that

$$
\begin{cases}\sum_{\mathbf{z}}(\mathbf{z}-\mathbf{x})^{\boldsymbol{\alpha}} b(\mathbf{x}, \mathbf{z})=\quad 0, \quad|\boldsymbol{\alpha}| \neq 2 \\ \sum_{\mathbf{z}}(\mathbf{z}-\mathbf{x})^{e_{u}+e_{v}} b(\mathbf{x}, \mathbf{z})= & x_{u}\left(\delta_{u v}-x_{v}\right), u, v=\overline{1, K}\end{cases}
$$

Then we obtain

$$
\begin{aligned}
\frac{\partial p(t, \mathbf{x}, \mathbf{y})}{\partial t} & =\sum_{\mathbf{z}} p(t, \mathbf{z}, \mathbf{y}) b(\mathbf{x}, \mathbf{z}) \\
& =\sum_{\mathbf{z}}\left\{\sum_{\boldsymbol{\alpha} \in \mathbb{N}_{0}^{K}} \frac{1}{\boldsymbol{\alpha} !} \frac{\partial^{\boldsymbol{\alpha}} p(t, \mathbf{x}, \mathbf{y})}{\partial \mathbf{x}^{\boldsymbol{\alpha}}}(\mathbf{z}-\mathbf{x})^{\boldsymbol{\alpha}}\right\} b(\mathbf{x}, \mathbf{z}) \\
& =\sum_{u, v=1}^{K} \frac{x_{u}\left(\delta_{u v}-x_{v}\right)}{2} \frac{\partial^{2} p(t, \mathbf{x}, \mathbf{y})}{\partial x_{u} \partial x_{v}}
\end{aligned}
$$

which is exactly the Wright-Fisher diffusion equation for $K+1$ alleles.

Now we shall prove that the master equation (2.1) yields the moment equations.

In fact, the $\boldsymbol{\alpha}-t h$ moment of this conditional probability function is

$$
m_{\boldsymbol{\alpha}}(t):=\sum_{\mathbf{j} \in \Omega_{K}^{2 N}}\left(\frac{\mathbf{j}}{2 N}\right)^{\boldsymbol{\alpha}} P(t, \mathbf{i}, \mathbf{j})
$$


Therefore we have

$$
\begin{aligned}
\dot{m}_{\boldsymbol{\alpha}}(t) & =\sum_{\mathbf{y}} \mathbf{y}^{\boldsymbol{\alpha}} \frac{\partial p(t, \mathbf{x}, \mathbf{y})}{\partial t} \frac{1}{2 N} \\
& =\sum_{\mathbf{y}} \mathbf{y}^{\boldsymbol{\alpha}} \sum_{\mathbf{z}} p(t, \mathbf{x}, \mathbf{z}) b(\mathbf{z}, \mathbf{y}) \frac{1}{2 N} \text { due to the Master equation (2.11) } \\
& =\sum_{\mathbf{z}}\left(\sum_{\mathbf{y}} \mathbf{y}^{\boldsymbol{\alpha}} b(\mathbf{z}, \mathbf{y})\right) p(t, \mathbf{x}, \mathbf{z}) \frac{1}{2 N} \\
& \left.=\sum_{\mathbf{z}}\left(-\frac{|\boldsymbol{\alpha}|(|\boldsymbol{\alpha}|-1)}{2} \mathbf{z}^{\boldsymbol{\alpha}}+\sum_{u=1}^{K} \frac{\alpha_{u}\left(\alpha_{u}-1\right)}{2} \mathbf{z}^{\boldsymbol{\alpha}-e_{u}}\right)\right) p(t, \mathbf{x}, \mathbf{z}) \frac{1}{2 N}
\end{aligned}
$$

by the formulae for $b$ from (2.14)

$$
=-\frac{|\boldsymbol{\alpha}|(|\boldsymbol{\alpha}|-1)}{2} m_{\boldsymbol{\alpha}}(t)+\sum_{u=1}^{K} \frac{\alpha_{u}\left(\alpha_{u}-1\right)}{2} m_{\boldsymbol{\alpha}-e_{u}}(t)
$$

\section{Moment Generating FUnCtions}

Definition 3.1. (1) Let $X$ be a random variable with discrete values with probability distribution function $p(x)=\mathbb{P}[X=x]$. The (exponential) moment generating function of the random variable $X$ is

$$
H(s):=\mathbb{E}\left[e^{s X}\right]=\sum_{x} e^{x s} p(x)
$$

(defined for those values of $s \in \mathbb{R}$ for which the sum converges).

(2) Let $\mathbf{X}=\left(X^{1}, \cdots, X^{K}\right)$ be a tuple of random variables with the joint probability distribution function $p\left(x^{1}, \ldots, x^{K}\right)=\mathbb{P}\left[X^{1}=x^{1}, \ldots, X^{K}=\right.$ $\left.x^{K}\right]$. The (exponential) moment generating function of $\mathbf{X}$ then is

$$
H\left(s_{1}, \ldots, s_{K}\right):=\mathbb{E}\left[e^{\sum^{K} s_{i} X^{i}}\right]=\sum_{x^{1}, \ldots, x^{K}} e^{\sum_{i} s_{i} x^{i}} p\left(x^{1}, \ldots, x^{K}\right)=\sum_{\mathbf{x}} e^{\mathbf{s} \cdot \mathbf{x}} p(\mathbf{x})
$$

(defined for those values of $\mathbf{s} \in \mathbb{R}^{K}$ for which the sum converges).

Here, the moments of $\mathbf{X}$ can directly be computed from the derivatives of $H(\mathbf{s})$ at $\mathbf{s}=0$,

$$
\mathbb{E}\left[\mathbf{X}^{\alpha}\right]=\left.\frac{\partial^{\alpha} H(\mathbf{s})}{\partial \mathbf{s}^{\alpha}}\right|_{\mathbf{s}=0} .
$$

We shall now derive the (second order) partial differential equation for the (exponential) moment generating functions of our Markov process $\mathbf{X}_{t}$. 
3.1. 2 alleles. The exponential moment generating function is

$$
\begin{aligned}
H(t ; s) & =\mathbb{E}\left[e^{s X_{t}}\right] \\
& =\sum_{n \geq 0} \frac{s^{n}}{n !} \mathbb{E}\left[\left(X_{t}\right)^{n}\right] \\
& =\sum_{n \geq 0} \frac{s^{n}}{n !} m_{n}(t),
\end{aligned}
$$

where $m_{n}(t)$ is the $n-t h$ moment of $X_{t}$ around 0 .

From the equation (2.10) for the moments

$$
\dot{m}_{n}(t)=-\frac{n(n-1)}{2} m_{n}(t)+\frac{n(n-1)}{2} m_{n-1}(t)
$$

we obtain

$$
\begin{aligned}
\frac{\partial H(t ; s)}{\partial t} & =\sum_{n \geq 0} \dot{m}_{n}(t) \frac{s^{n}}{n !} \\
& =\sum_{n \geq 0}\left[-\frac{n(n-1)}{2} m_{n}(t)+\frac{n(n-1)}{2} m_{n-1}(t)\right] \frac{s^{n}}{n !} \\
& =\sum_{n \geq 2}-\frac{1}{2} m_{n}(t) \frac{s^{n}}{(n-2) !}+\sum_{n \geq 2} \frac{1}{2} m_{n-1}(t) \frac{s^{n}}{(n-2) !} \\
& =-\frac{s^{2}}{2} \sum_{n \geq 0} m_{n+2}(t) \frac{s^{n}}{n !}+\frac{s^{2}}{2} \sum_{n \geq 0} m_{n+1}(t) \frac{s^{n}}{n !} \\
& =-\frac{s^{2}}{2} \frac{\partial^{2}}{\partial s^{2}} H(t ; s)+\frac{s^{2}}{2} \frac{\partial}{\partial s} H(t ; s) .
\end{aligned}
$$

We now consider solutions of such equations. First, we solve the equation (3.2) by separation of variables. With $H(t ; s)=T(t) S(s)$, the equation becomes

$$
\frac{T^{\prime}(t)}{T(t)}=\frac{-s^{2} S^{\prime \prime}(s)+s^{2} S^{\prime}(s)}{2 S}=-\lambda .
$$

It follows that $T(t)=C e^{-\lambda t}$ and $S(s)$ satisfies the ODE

$$
-x^{2} y_{x x}+x^{2} y_{x}=-2 \lambda y \text {. }
$$

By putting $y(x)=\sum_{n \geq 0} a_{n} x^{n}$ and equating coefficients in the ODE (3.3) we obtain:

(1) If

$$
\lambda \notin \Lambda:=\left\{\mu_{n}=\frac{n(n-1)}{2}, n \in \mathbb{N}\right\}
$$

then the ODE (3.3) has a unique solution $y(x)=0$;

(2) If $\lambda=\mu_{0}$ then $y_{0}(x)=a_{0}^{(0)}:=1$; 
(3) If $\lambda=\mu_{k}$ for some $k \geq 1$ then the solution is of the form

$$
y_{k}(x)=\sum_{n \geq 0} a_{n}^{(k)} x^{n}
$$

where

$$
a_{n}^{(k)}=\left\{\begin{array}{l}
0, \quad \text { if } n<k \\
1, \quad \text { if } n=k \\
\frac{n-1}{2\left(\mu_{n}-\mu_{k}\right)} \cdots \frac{k}{2\left(\mu_{k+1}-\mu_{k}\right)}, \quad \text { if } n \geq k+1 .
\end{array}\right.
$$

Therefore the solution of (3.2) is

$$
\begin{aligned}
H(t ; s) & =\sum_{k \geq 0} c_{k} y_{k}(s) e^{-\mu_{k} t} \\
& =\sum_{k \geq 0} c_{k}\left(\sum_{n \geq k} a_{n}^{(k)} s^{n}\right) e^{-\mu_{k} t} \\
& =\sum_{n \geq 0}\left(n ! \sum_{k=0}^{n} c_{k} a_{n}^{(k)} e^{-\mu_{k} t}\right) \frac{s^{n}}{n !} .
\end{aligned}
$$

This yields the moment formula

$$
m_{n}(t)=n ! \sum_{k=0}^{n} c_{k} a_{n}^{(k)} e^{-\mu_{k} t}=\sum_{k=0}^{n} c_{k} A_{n}^{(k)} e^{-\mu_{k} t} .
$$

The coefficients $c_{k}$ can be calculated from the initial condition

$$
\left(\frac{i}{2 N}\right)^{n}:=p^{n}=m_{n}(0)=\sum_{k=0}^{n} c_{k} A_{n}^{(k)}, \quad \forall n \geq 0 .
$$

In fact, by representing these equalities in matrix form

$$
\left[\begin{array}{ccccc}
1 & 0 & \cdots & 0 & 0 \\
A_{1}^{(0)} & 1 & \cdots & 0 & 0 \\
\vdots & \vdots & \ddots & \vdots & \\
A_{n-1}^{(0)} & A_{n-1}^{(1)} & \cdots & (n-1) ! & 0 \\
A_{n}^{(0)} & A_{n}^{(1)} & \cdots & A_{n}^{(n-1)} & n !
\end{array}\right]\left[\begin{array}{c}
c_{0} \\
c_{1} \\
\vdots \\
c_{n-1} \\
c_{n}
\end{array}\right]=\left[\begin{array}{c}
1 \\
p \\
\vdots \\
p^{n-1} \\
p^{n}
\end{array}\right]
$$

it follows that

$$
\left[\begin{array}{c}
c_{0} \\
c_{1} \\
\vdots \\
c_{n-1} \\
c_{n}
\end{array}\right]=\left[\begin{array}{ccccc}
1 & 0 & \cdots & 0 & 0 \\
A_{1}^{(0)} & 1 & \cdots & 0 & 0 \\
\vdots & \vdots & \ddots & \vdots & \\
A_{n-1}^{(0)} & A_{n-1}^{(1)} & \cdots & (n-1) ! & 0 \\
A_{n}^{(0)} & A_{n}^{(1)} & \cdots & A_{n}^{(n-1)} & n !
\end{array}\right]^{-1}\left[\begin{array}{c}
1 \\
p \\
\vdots \\
p^{n-1} \\
p^{n}
\end{array}\right]
$$


Remark 3.2. We can easily check some instances: Because of $c_{0}=1 ; c_{1}=$ $p ; c_{2}=\frac{p^{2}-p}{2} ; c_{3}=\frac{p^{3}-3 / 2 p^{2}+1 / 2 p}{6}$ then $m_{0}(t)=1 ; m_{1}(t)=p ; m_{2}(t)=p+\left(p^{2}-\right.$ $p) e^{-t} ; m_{3}(t)=p+3 / 2\left(p^{2}-p\right) e^{-t}+\left(p^{3}-3 / 2 p^{2}+1 / 2 p\right) e^{-3 t}$.

This also yields the fixation probability at time $t$ is (see also [10])

$$
\begin{aligned}
P(t, i, 2 N) & =\lim _{n \rightarrow \infty} m_{n}(t) \\
& =\lim _{n \rightarrow \infty} \sum_{k=0}^{n} c_{k} A_{n}^{(k)} e^{-\mu_{k} t} \\
& =p+\lim _{n \rightarrow \infty} \sum_{k=2}^{n} c_{k} A_{n}^{(k)} e^{-\mu_{k} t},
\end{aligned}
$$

and the eventual fixation probability

$$
\begin{aligned}
P(\infty, i, 2 N) & =\lim _{t \rightarrow \infty}\left(p+\lim _{n \rightarrow \infty} \sum_{k=2}^{n} c_{k} A_{n}^{(k)} e^{-\mu_{k} t}\right) \\
& =p+\lim _{n \rightarrow \infty} \lim _{t \rightarrow \infty} \sum_{k=2}^{n} c_{k} A_{n}^{(k)} e^{-\mu_{k} t} \\
& =p .
\end{aligned}
$$

Similarly, by calculating for the other allele $\left(Y_{t}=1-X_{t}\right)$, we obtain the extinction probability at time $t$ is (see also [10])

$$
\begin{aligned}
P(t, i, 0) & =\lim _{n \rightarrow \infty} m_{n}^{\prime}(t) \\
& =1-p+\lim _{n \rightarrow \infty} \sum_{k=0}^{n} c_{k}^{\prime} A_{n}^{(k)} e^{-\mu_{k} t} \\
& =1-p+\lim _{n \rightarrow \infty} \sum_{k=2}^{n} c_{k}^{\prime} A_{n}^{(k)} e^{-\mu_{k} t},
\end{aligned}
$$

and the eventual extinction probability

$$
\begin{aligned}
P(\infty, i, 0) & =\lim _{t \rightarrow \infty}\left(1-p+\lim _{n \rightarrow \infty} \sum_{k=2}^{n} c_{k} A_{n}^{(k)} e^{-\mu_{k} t}\right) \\
& =1-p .
\end{aligned}
$$

The moments of the sojourn and absorption times were derived by Nagylaki [9] for two alleles, and by Lessard and Lahaie [8] in the multi-allele case. We denote by $T_{n+1}^{k+1}(p)=\inf \left\{t>0: X_{t} \in \bar{V}_{k} \mid X_{0}=p\right\}$ the first time when the population has (at most) $k+1$ alleles. $T_{n+1}^{k+1}(p)$ is a continuous random variable valued in $[0, \infty)$ and we denote by $\phi(t, p)$ its probability density function. It is easy to see that $\bar{V}_{k}$ is invariant under the process $\left(X_{t}\right)_{t \geq 0}$, i.e. if $X_{s} \in \bar{V}_{k}$ then $X_{t} \in \bar{V}_{k}$ for all $t \geq s$ (once an allele is lost from the 
population, it can never again be recovered). We have the equality

$$
\mathbb{P}\left(T_{2}^{1}(p) \leq t\right)=P(t, i, 0)+P(t, i, 2 N)
$$

Therefore the expectation of the absorption time is

$$
\begin{aligned}
\mathbb{E}\left(T_{2}^{1}(p)\right) & =\int_{0}^{\infty} t \frac{\partial}{\partial t}(P(t, i, 0)+P(t, i, 2 N)) d t \\
& =-\lim _{n \rightarrow \infty} \sum_{k=2}^{n}\left(c_{k}+c_{k}^{\prime}\right) A_{n}^{(k)} \frac{1}{\mu_{k}} .
\end{aligned}
$$

Moreover we have

$$
\sum_{j=0}^{2 N}\left(\frac{j}{2 N}\right)^{n} P(\infty, i, j)=\lim _{t \rightarrow \infty} m_{n}(t)= \begin{cases}p, & \text { for } n \geq 1 \\ 1, & \text { for } n=0\end{cases}
$$

Therefore we obtain the eventually probability

$$
P(\infty, i, j)=p \delta_{2 N, j}+(1-p) \delta_{0, j}
$$

The probability of heterogeneity is (also see [10])

$$
\begin{aligned}
H_{t} & :=2 \sum_{j=0}^{2 N} \frac{j}{2 N}\left(1-\frac{j}{2 N}\right) P(t, i, j) \\
& =2\left(m_{1}(t)-m_{2}(t)\right) \\
& =2\left(p-\left(p+c_{2} A_{2}^{(2)} e^{-t}\right)\right) \\
& =2 p(1-p) e^{-t} .
\end{aligned}
$$

3.2. $K+1$ alleles. We can apply the same scheme for any $K$. The exponential generating function now is

$$
H\left(t ; s_{1}, \ldots, s_{K}\right)=\sum_{\boldsymbol{\alpha}} m_{\boldsymbol{\alpha}}(t) \frac{\mathbf{s}^{\boldsymbol{\alpha}}}{\boldsymbol{\alpha} !},
$$

where $m_{\boldsymbol{\alpha}}(t)$ is the $\boldsymbol{\alpha}-t h$ moment of $\mathbf{X}_{t}$ around 0 .

From (2.17), i.e.,

$$
\dot{m}_{\boldsymbol{\alpha}}(t)=-\frac{|\boldsymbol{\alpha}|(|\boldsymbol{\alpha}|-1)}{2} m_{\boldsymbol{\alpha}}(t)+\sum_{i=1}^{K} \frac{\alpha_{i}\left(\alpha_{i}-1\right)}{2} m_{\boldsymbol{\alpha}-e_{i}}(t),
$$


we obtain

$$
\begin{aligned}
\frac{\partial H(t ; \mathbf{s})}{\partial t}= & \sum_{\boldsymbol{\alpha}} \dot{m}_{\boldsymbol{\alpha}}(t) \frac{\mathbf{s}^{\boldsymbol{\alpha}}}{\boldsymbol{\alpha} !} \\
= & \sum_{\boldsymbol{\alpha}}\left[-\frac{|\boldsymbol{\alpha}|(|\boldsymbol{\alpha}|-1)}{2} m_{\boldsymbol{\alpha}}(t)+\sum_{i=1}^{K} \frac{\alpha_{i}\left(\alpha_{i}-1\right)}{2} m_{\boldsymbol{\alpha}-e_{i}}(t)\right] \frac{\mathbf{s}^{\boldsymbol{\alpha}}}{\boldsymbol{\alpha} !} \\
= & \sum_{\boldsymbol{\alpha}}\left(-\frac{\sum_{i \neq j} \alpha_{i} \alpha_{j}}{2}-\frac{\sum_{i} \alpha_{i}\left(\alpha_{i}-1\right)}{2}\right) m_{\boldsymbol{\alpha}}(t) \frac{\mathbf{s}^{\boldsymbol{\alpha}}}{\boldsymbol{\alpha} !} \\
& +\sum_{\boldsymbol{\alpha}} \sum_{i=1}^{K} \frac{\alpha_{i}\left(\alpha_{i}-1\right)}{2} m_{\boldsymbol{\alpha}-e_{i}}(t) \frac{\mathbf{s}^{\boldsymbol{\alpha}}}{\boldsymbol{\alpha} !} \\
= & -\frac{1}{2} \sum_{i \neq j} s_{i} s_{j} \frac{\partial^{2} H(t, \mathbf{s})}{\partial s_{i} \partial s_{j}}-\frac{1}{2} \sum_{i} s_{i}^{2} \frac{\partial^{2} H(t, \mathbf{s})}{\partial s_{i}^{2}}+\sum_{i=1}^{K} \frac{1}{2} \sum_{i} s_{i}^{2} \frac{\partial H(t, \mathbf{s})}{\partial s_{i}} \\
= & -\frac{1}{2} \sum_{i, j=1}^{K} s_{i} s_{j} \frac{\partial^{2}}{\partial s_{i} \partial s_{j}} H(t ; \mathbf{s})+\sum_{i=1}^{K} \frac{s_{i}^{2}}{2} \frac{\partial}{\partial s_{i}} H(t ; \mathbf{s})
\end{aligned}
$$

Separating variables as above, $T(t)=C e^{-\lambda t}$ and $S(\mathbf{s})$ satisfies the PDE

$$
-\frac{1}{2} \sum_{i, j=1}^{K} s_{i} s_{j} \frac{\partial^{2}}{\partial s_{i} \partial s_{j}} y(\mathbf{s})+\sum_{i=1}^{K} \frac{s_{i}^{2}}{2} \frac{\partial}{\partial s_{i}} y(\mathbf{s})=-\lambda y(\mathbf{s}) .
$$

By putting $y(\mathbf{s})=\sum_{\boldsymbol{\alpha}} a_{\boldsymbol{\alpha}} \mathbf{s}^{\boldsymbol{\alpha}}$ and equating coefficients in the PDE (3.17) we obtain:

(1) If

$$
\lambda \notin \Lambda:=\left\{\mu_{n}=\frac{n(n-1)}{2}, n \in \mathbb{N}\right\}
$$

then the PDE (3.17) has a unique solution $y(\mathbf{s})=0$;

(2) If $\lambda=\mu_{0}$ then $y_{0}(x)=a_{0}^{(0)}:=1$;

(3) If $\lambda=\mu_{k}$ for some $k \geq 1$ then there are $\left(\begin{array}{l}k \\ 2\end{array}\right)$ independent solutions of the form

$$
y_{k, \boldsymbol{\alpha}}(\mathbf{s})=\sum_{\boldsymbol{\beta}} a_{\boldsymbol{\alpha}, \boldsymbol{\beta}}^{(k)} \mathbf{s}^{\boldsymbol{\beta}}, \quad \forall|\boldsymbol{\alpha}|=k .
$$

where

$$
a_{\boldsymbol{\alpha}, \boldsymbol{\beta}}^{(k)}=\left\{\begin{array}{l}
0, \quad \text { if }|\boldsymbol{\beta}|<k \\
\delta_{\boldsymbol{\beta}}^{\boldsymbol{\alpha}}, \quad \text { if }|\boldsymbol{\beta}|=k \\
\text { inductively defined by }(\underline{3.200}) \text { below, if }|\boldsymbol{\beta}| \geq k+1
\end{array}\right.
$$




$$
a_{\boldsymbol{\alpha}, \boldsymbol{\beta}}^{(k)}=\frac{\sum_{i=1}^{K}\left(\beta_{i}-1\right) a_{\boldsymbol{\alpha}, \boldsymbol{\beta}-e_{i}}^{(k)}}{|\boldsymbol{\beta}|(|\boldsymbol{\beta}|-1)-k(k-1)} .
$$

Therefore the solution of equation (3.17) is

$$
\begin{aligned}
H(t ; \mathbf{s}) & =\sum_{k \geq 0} \sum_{|\boldsymbol{\alpha}|=k} c_{k, \boldsymbol{\alpha}} y_{k, \boldsymbol{\alpha}}(\mathbf{s}) e^{-\mu_{k} t} \\
& =\sum_{k \geq 0} \sum_{|\boldsymbol{\alpha}|=k} c_{k, \boldsymbol{\alpha}}\left(\sum_{\boldsymbol{\beta}} a_{\boldsymbol{\alpha}, \boldsymbol{\beta}^{(} \mathbf{s}^{\boldsymbol{\beta}}}^{(k)}\right) e^{-\mu_{k} t} \\
& =\sum_{\boldsymbol{\beta}}\left(\sum_{k=0}^{|\boldsymbol{\beta}|} \sum_{|\boldsymbol{\alpha}|=k} c_{k, \boldsymbol{\alpha}} a_{\boldsymbol{\alpha}, \boldsymbol{\beta}}^{(k)} e^{-\mu_{k} t}\right) \mathbf{s}^{\boldsymbol{\beta}} \\
& =\sum_{\boldsymbol{\beta}} \boldsymbol{\beta} !\left(\sum_{|\boldsymbol{\alpha}| \leq|\boldsymbol{\beta}|} c_{|\boldsymbol{\alpha}|, \boldsymbol{\alpha}} a_{\boldsymbol{\alpha}, \boldsymbol{\beta}}^{(|\boldsymbol{\alpha}|)} e^{-\mu_{k} t}\right) \frac{\mathbf{s}^{\boldsymbol{\beta}}}{\boldsymbol{\beta} !}
\end{aligned}
$$

This yields the moment formula

$$
m_{\boldsymbol{\beta}}(t)=\boldsymbol{\beta} ! \sum_{|\boldsymbol{\alpha}| \leq|\boldsymbol{\beta}|} c_{|\boldsymbol{\alpha}|, \boldsymbol{\alpha}} a_{\boldsymbol{\alpha}, \boldsymbol{\beta}}^{(|\boldsymbol{\alpha}|)} e^{-\mu_{k} t}
$$

where the coefficients $c_{|\boldsymbol{\alpha}|, \boldsymbol{\alpha}}$ can be computed from the initial condition

$$
p^{\boldsymbol{\beta}}=m_{\boldsymbol{\beta}}(0)=\boldsymbol{\beta} ! \sum_{|\boldsymbol{\alpha}| \leq|\boldsymbol{\beta}|} c_{|\boldsymbol{\alpha}|, \boldsymbol{\alpha}} a_{\boldsymbol{\alpha}, \boldsymbol{\beta}}^{(|\boldsymbol{\alpha}|)}, \quad \forall \boldsymbol{\beta}
$$

\section{ACKNOWLEDGEMENT}

The research leading to these results has received funding from the European Research Council under the European Union Seventh Framework Programme (FP7/2007-2013)/ERC grant agreement no. 267087.

\section{REFERENCES}

[1] Warren J. Ewens, Mathematical Population Genetics I. Theoretical Introduction, Springer-Verlag New York Inc., Interdisciplinary Applied Mathematics, 2nd ed., 2004.

[2] Fisher R. A., On the dominance ratio, Proc. Roy. Soc. Edinb., 42 (1922), 321-341.

[3] Gardiner C.W., Handbook of Stochastic methods: For physics, Chemistry and the Natural Sciences, Springer, New York (2004).

[4] Houchmandzadeh B., Vallade M., Alternative to the diffusion equation in population genetics, Physical Review E. 82 (2010), 051913.

[5] Jost J., Mathematical Methods in Biology and Neurobiology, Springer, 2014

[6] Kimura M., Solution of a Process of Random Genetic Drift with a Continuous Model, PNAS-USA, Vol. 41, No. 3, (1955), 144-150.

[7] Kimura M., Random genetic drift in multi-allele locus, Evolution, 9 (1955), 419435. 
[8] Lessard S., Lahaie P., Fixation probability with multiple alleles and projected average allelic effect on selection, Theoretical Population Biology 75 (2009), 266277

[9] Nagylaki T., The Decay of Genetic Variability in Geographically Structured Populations, PNAS 71 (1974), 2932-2936.

[10] Tran T.D., Hofrichter J., Jost J., An introduction to the mathematical structure of the Wright-Fisher model of population genetics, Theory Biosc. 132 (2013), 73-82.

[11] Tran T.D., Hofrichter J., Jost J., A general solution of the Wright-Fisher model of random genetic drift, Preprint

[12] S. Wright, Evolution in Mendelian populations, Genetics, 16 (1931), 97-159.

Tat Dat Tran, Max Planck Institute for Mathematics in the Sciences, Inselstrasse 22, 04103 Leipzig, Germany, trandat@mis.mpg.de

Julian Hofrichter, Max Planck Institute for Mathematics in the Sciences, Inselstrasse 22, 04103 Leipzig, Germany, julian.hofrichter@mis.mpg.de

Jürgen Jost, Max Planck Institute for Mathematics in the Sciences, Inselstrasse 22, 04103 Leipzig, Germany

Santa Fe Institute for the Sciences of Complexity, Santa Fe, NM 87501, USA, jost@mis.mpg.de 